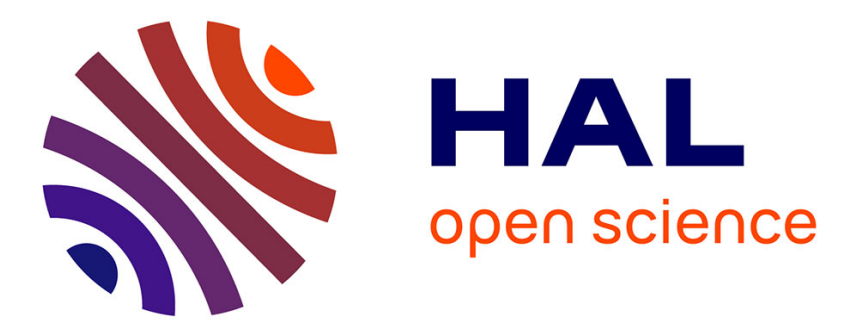

\title{
Strategies of locomotor collision avoidance
}

Patrizia Basili, Murat Sağlam, Thibault Kruse, Markus Huber, Alexandra

Kirsch, Stefan Glasauer

\section{To cite this version:}

Patrizia Basili, Murat Sağlam, Thibault Kruse, Markus Huber, Alexandra Kirsch, et al.. Strategies of locomotor collision avoidance. Gait \& Posture, 2013, 37 (3), pp.385 - 390. 10.1016/j.gaitpost.2012.08.003 . hal-01684297

\section{HAL Id: hal-01684297 \\ https://hal.science/hal-01684297}

Submitted on 15 Jan 2018

HAL is a multi-disciplinary open access archive for the deposit and dissemination of scientific research documents, whether they are published or not. The documents may come from teaching and research institutions in France or abroad, or from public or private research centers.
L'archive ouverte pluridisciplinaire HAL, est destinée au dépôt et à la diffusion de documents scientifiques de niveau recherche, publiés ou non, émanant des établissements d'enseignement et de recherche français ou étrangers, des laboratoires publics ou privés. 
NOTICE: this is the author's version of a work that was accepted for publication in Gait \& Posture . Changes resulting from the publishing process, such as peer review, editing, corrections, structural formatting, and other quality control mechanisms may not be reflected in this document. Changes may have been made to this work since it was submitted for publication. A definitive version was subsequently published in Gait \& Posture: http://dx.doi.org/10.1016/j.gaitpost.2012.08.003, published online 12 September 2012

\title{
Strategies of Collision Avoidance
}

\author{
Patrizia Basilia, Murat Sağlamª,b, Thibault Krusec, Markus Huber ${ }^{a}$, \\ Alexandra Kirsch ${ }^{d}$, Stefan Glasauer ${ }^{a, b, *}$
}

\author{
${ }^{a}$ Center for Sensorimotor Research, Ludwig-Maximilian University Munich, Germany \\ ${ }^{b}$ German Dizziness Center IFB, Ludwig-Maximilian University, Munich, Germany \\ 'Intelligent Autonomous Systems Group Department of Informatics, Technische Universität München \\ ${ }^{d}$ Department of Computer Science, University of Tübingen, Tübingen, Germany
}

\section{Abstract}

Collision avoidance during locomotion can be achieved by a variety of strategies. While in some situations only a single trajectory will successfully avoid impact, in many cases several different strategies are possible. Locomotor experiments in the presence of static boundary conditions have suggested that the choice of an appropriate trajectory is based on a maximum-smoothness strategy. Here we analyzed locomotor trajectories of subjects avoiding collision with another human crossing their path orthogonally. In such a case, changing walking direction while keeping speed or keeping walking direction while changing speed would be two extremes of solving the problem. Our participants clearly favored changing their walking speed while keeping the path on a straight line between start and goal. To interpret this result, we calculated the costs of the chosen trajectories in terms of a smoothness-maximization criterion and simulated the trajectories with a computational model. Data analysis together with model simulation showed that the experimentally chosen trajectory to avoid collision with a moving human is not the optimally smooth solution. However, even though the trajectory is not globally

\footnotetext{
* Corresponding author at: Center for Sensorimotor Research, Marchioninistr 23, 81377 Munchen, Germany. E-mail addresses: s.glasauer@Irz.uni-muenchen.de, sglasauer@nefo.med.uni-muenchen.de (S. Glasauer).
} 
smooth, it was still locally smooth. Modeling further confirmed that, in presence of the moving human, there is always a trajectory that would be smoother but would deviate from the straight line. We therefore conclude that the maximum smoothness strategy previously suggested for static environments no longer holds for locomotor path planning and execution in dynamically changing environments.

\section{Introduction}

On our way back from work, we cross path with dozens, maybe even hundreds of people, but hardly ever we experience a collision with someone else. Our ability to infer the others' intentions from action observation (for review, see [1]) apparently enables us to rapidly recognize others as being potential obstacles and predict their future path. Consequently we are able to adapt our own motion in order to avoid collisions. However, there are infinitely many possible solutions to avoid collision with a moving obstacle [2]. The analysis of whole body motion in the presence of static obstacles (e.g., $[3,4]$ ) or boundary conditions has shown that walking trajectories, just like arm movements, are stereotypical under the repeated conditions $[5,6]$. This suggests that humans use a specific strategy to solve the problem of trajectory formation for goal-directed locomotion, i.e., walking from one location to another. Previous studies have proposed that humans minimize a 'cost' associated with a trajectory and that this minimization is equivalent to a maximumsmoothness strategy $[7,8]$.

Whether this strategy also holds for collision avoidance is yet unknown and investigations in the presence of moving obstacles are relatively rare $[9,10]$. Whereas some studies have analyzed how visual information affects perception of the others' movement $[3,11]$ and of obstacles $[12,13]$, several investigations on human locomotion behavior focused on average behavior of crowds rather than single agents (e.g., [14-16]). For human-aware robot control, which also faces the problem of obstacle avoidance (e.g., [17]), a common strategy, often based on the 
concept of 'proxemics' [18], has been adopted for different cases of obstacle avoidance and types of interactions [19]. While static obstacles can easily be circumvented by a global path planning procedure, the exact trajectory of moving obstacles cannot be predicted without error and thus may require local planning, i.e., feedback about the current location or motion of the obstacle has to be incorporated to initiate re-planning and corrections.

In the current work, we first asked whether collision avoidance in the presence of another moving agent results in stereotypical trajectories, which would suggest a predefined strategy. We then analyzed collision avoidance under different conditions in order to clarify whether minimization of smoothness costs adequately describes the experimental findings. In order to disentangle different possibilities of trajectory formation, we devised a simple model using a maximum-smoothness constraint $[20,21]$ and simulated our experimental conditions.

\section{Materials and Methods}

\section{Experiments}

13 subjects (age 25-43, 7 females) participated in the experiments. Two persons, an interferer (male, 37 years old) and one subject walked from a predefined starting position to a fixed goal position. The starting angle between the two intended paths was $90^{\circ}$ and the starting positions were at the same distance from the intersection (Fig. 1A). Using a motion tracking system (IS-600 Mark 2, InterSense Inc., USA), the head position of the subject was tracked using a 6-DOF sensor mounted on a helmet (Fig. 1B) using infrared and ultrasound signals at $150 \mathrm{~Hz}$ and the head position of the interferer was tracked with a wireless sensor at $20-50 \mathrm{~Hz}$. The size of the tracked area was $4 \mathrm{~m} \times 4 \mathrm{~m}$ in the middle of a room of $38 \mathrm{~m}^{2}$. All experiments were done with open eyes and natural lighting conditions. The interferer and the subjects were instructed before the experiment about their task (see below) and told not to communicate verbally. Subjects gave their written consent prior to the experiment 
according to the declaration of Helsinki.

Four different conditions were tested for each subject in 11 consecutive trials: (1: None) Subject walked alone from the start to the end position without obstacle (4 trials); (2: Moving) Subject and interferer started to walk at a common starting signal (4 consecutive trials); (3: Catch) Subject and interferer walked simultaneously but the interferer stopped unexpectedly at the intersection with the subject's path (one trial per subject); (4: Retest) Scenario (2) was repeated again two times. In the Moving obstacle condition, the interferer was instructed not to consider the behavior of or look at the subject, but to try being the first to pass. The subject was informed that the interferer would not react to the subject. Condition (3), the catch trial, was used to assess on-line correction strategies when an unexpected event/obstacle occurs. In condition (4) the scenario presented in (2) was repeated to test whether the previous catch trial influenced the behavior of the subject.

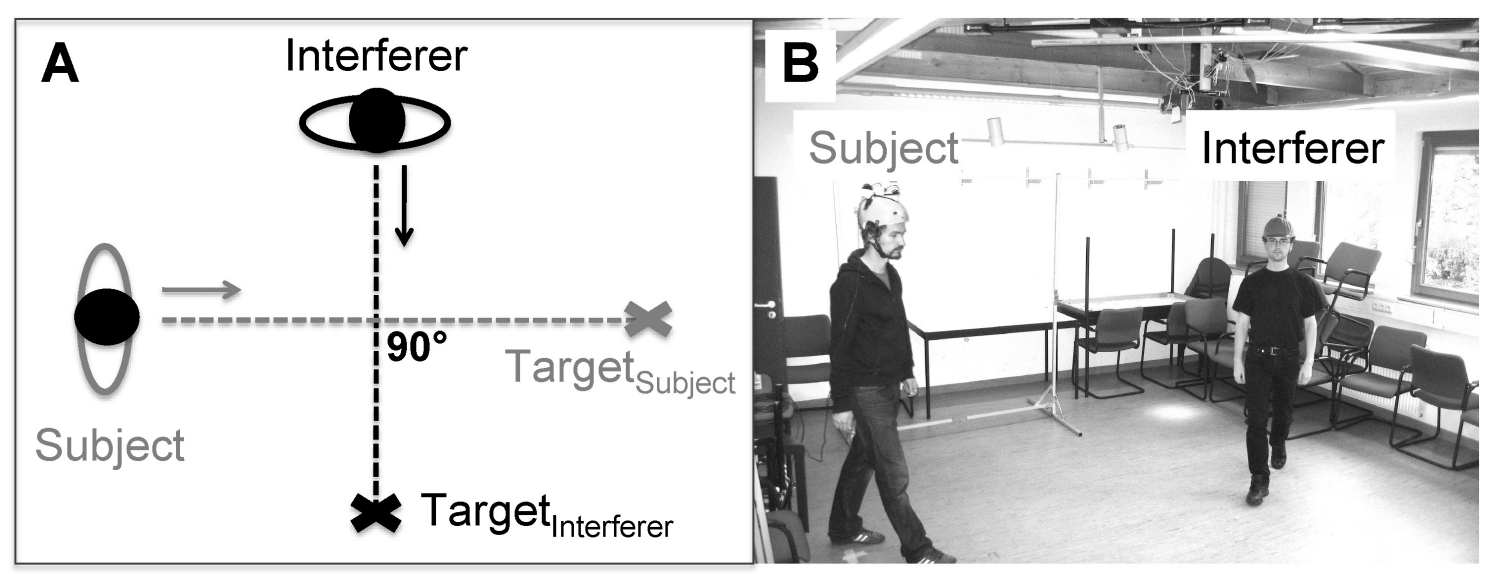

Figure 1: Experimental Setup. Subject and Interferer were instructed to reach a pre-defined target position. At an acoustic signal, both participants started to walk from initial positions at an equal distance to the intersection point of their paths. The interferer was instructed to pass before the subject and avoid gaze contact. A: Overhead view of the experimental setup. B: Experimental situation.

\section{Data analysis}

Raw data were analyzed using Matlab (The Mathworks, Natick, MA). 3D position data were filtered using a Gaussian lowpass filter (cutoff frequency $2 \mathrm{~Hz}$ ). The 
resulting trajectory was differentiated to yield 3D velocity. To define start and end of a movement, a velocity criterion of $20 \%$ of the maximum speed was used. Velocity profiles were normalized before averaging. Smoothness of the trajectory is quantified by the integrated squared jerk and the cost $J$ is computed as follows [20]:

$$
J=\int_{0}^{t_{E}} x(t)^{2}+\cdots(t)^{2} d t
$$

where $(x, y)$ are the Cartesian coordinates of the subject position and $t_{E}$ is the movement duration. Statistical analysis was performed using the Statistics Toolbox of Matlab. The significance level was set to a p-value of 0.05 .

\section{Simulation of Human Walking Behavior}

We simulated the subject's movement by minimizing the jerk of the movement in the presence of a pre-defined interferer movement. Minimum jerk movement was computed given the velocity and position at the two end points using the algorithm presented in [21]. In addition to end-point constraints, we defined one via-point on the path to account for the path planning of the subject. Time of passage, position, and velocity at the via-point were obtained from the experimental velocity profile. Thus, the simulated movement profile was the minimum jerk movement given the particular via- and end-point constraints. Simulations were performed using the Matlab software (The Mathworks, Natick, MA) and using the procedure explained in [21].

\section{Results}

\section{Experiments}

In general, trajectories were very stereotyped both within and between subjects demonstrating that the same strategy was used in each trial of one condition. Exceptions were seen only in a few cases, which are described below together with the respective results for each condition. Table 1 gives an overview of several 
descriptive variables per condition including the average cost of the trajectory in terms of a minimum-jerk strategy (see Methods).

\begin{tabular}{|c|c|c|c|c|c|}
\hline Condition & $\begin{array}{l}\text { Length of } \\
\text { trajectory [m] }\end{array}$ & $\begin{array}{l}\text { Max deviation } \\
{[\mathrm{m}]}\end{array}$ & $\begin{array}{l}\text { Mean peak } \\
\text { velocity }[\mathrm{m} / \mathrm{s}]\end{array}$ & $\begin{array}{l}\text { Mean duration until } \\
\text { peak velocity [s] }\end{array}$ & $\begin{array}{l}\text { Jerk cost } \\
{\left[\mathrm{m}^{2} / \mathrm{s}^{5}\right]}\end{array}$ \\
\hline $\begin{array}{l}\text { 1. none } \\
\text { 2. moving }\end{array}$ & $\begin{array}{l}3.17 \pm 0.12 \\
3.09 \pm 0.16\end{array}$ & $\begin{array}{l}0.3 \pm 0.07 \\
0.23 \pm 0.04\end{array}$ & $\begin{array}{l}0.97 \pm 0.34 \\
\text { (a) } 0.67 \pm 0.06\end{array}$ & $\begin{array}{l}2.29 \pm 0.42 \\
\text { (a) } 1.32 \pm 0.16\end{array}$ & $\begin{array}{l}3.48 \pm 1.61 \\
7.54 \pm 4.74\end{array}$ \\
\hline 3. catch & $3.36 \pm 0.19$ & $0.43 \pm 0.15$ & $\begin{array}{l}\text { (b) } 0.82 \pm 0.06 \\
\text { (a) } 0.65 \pm 0.1\end{array}$ & $\begin{array}{l}\text { (b) } 3.5 \pm 0.3 \\
\text { (a) } 1.31 \pm 0.23\end{array}$ & $37.9 \pm 30.4$ \\
\hline 4. retest & $3.03 \pm 0.36$ & $0.26 \pm 0.05$ & $\begin{array}{l}\text { (b) } 0.81 \pm 0.11 \\
\text { (a) } 0.66 \pm 0.08\end{array}$ & $\begin{array}{l}\text { (b) } 4.64 \pm 0.71 \\
\text { (a) } 1.44 \pm 0.24\end{array}$ & $7.17 \pm 3.9$ \\
\hline & & & (b) $0.61 \pm 0.07$ & (b) $2.04 \pm 0.31$ & \\
\hline
\end{tabular}

Table 1: Average and standard deviation of descriptive variables for each condition. In conditions 2, 3 , and 4, two velocity peaks ( $a$ and b) were observed. For conditions 2 and 3, alsothe average walking velocity of the interferer is given (i).

None: When walking towards the predefined target position without obstacle, subjects performed stereotyped straight trajectories (Fig. 2A) with a typical bellshaped velocity profile (Fig. 3A). As expected, overall costs were low in this condition (Table 1).

Moving: In this scenario, subject and interferer started walking simultaneously. While the interferer reached his target position without considering the other person, the subject was forced to adapt her/his motion to avoid a collision. The strategy adopted by the subjects was to keep a straight path as if walking alone (condition 1, None) but regulate walking speed (Fig. 2B, 3B). The subjects braked and accelerated again before the interferer had reached the possible collision position (intersection of both paths). The two peaks in the velocity profile (Fig. 3B) occurred for all subjects except one, who exhibited an alternative strategy using a slow acceleration phase. A collision between subject and interferer occurred only in a single trial of one subject. The overall movement cost was significantly larger than in condition 1 (paired t-test, $p=0.03$ ).

Catch: When the interferer unexpectedly stopped in the middle of the subject's path, the subject had to adapt the trajectory in order to avoid a collision with the interferer. This path re-planning induced a rapid braking (Fig. 2C) together with a modification of the trajectory (Fig. 3C). All subjects took a route behind the standing 
interferer. The high cost of the movement reflects the non-optimal trajectory chosen due to the unexpected obstacle behavior. The costs were significantly larger than in the moving condition $(p=0.008)$.

Retest: Even the first trial after the catch trial did not show any difference to the trials before (except for one subject who changed the strategy by adapting the path). Movement cost was therefore not different to the moving case $(p=0.23)$.
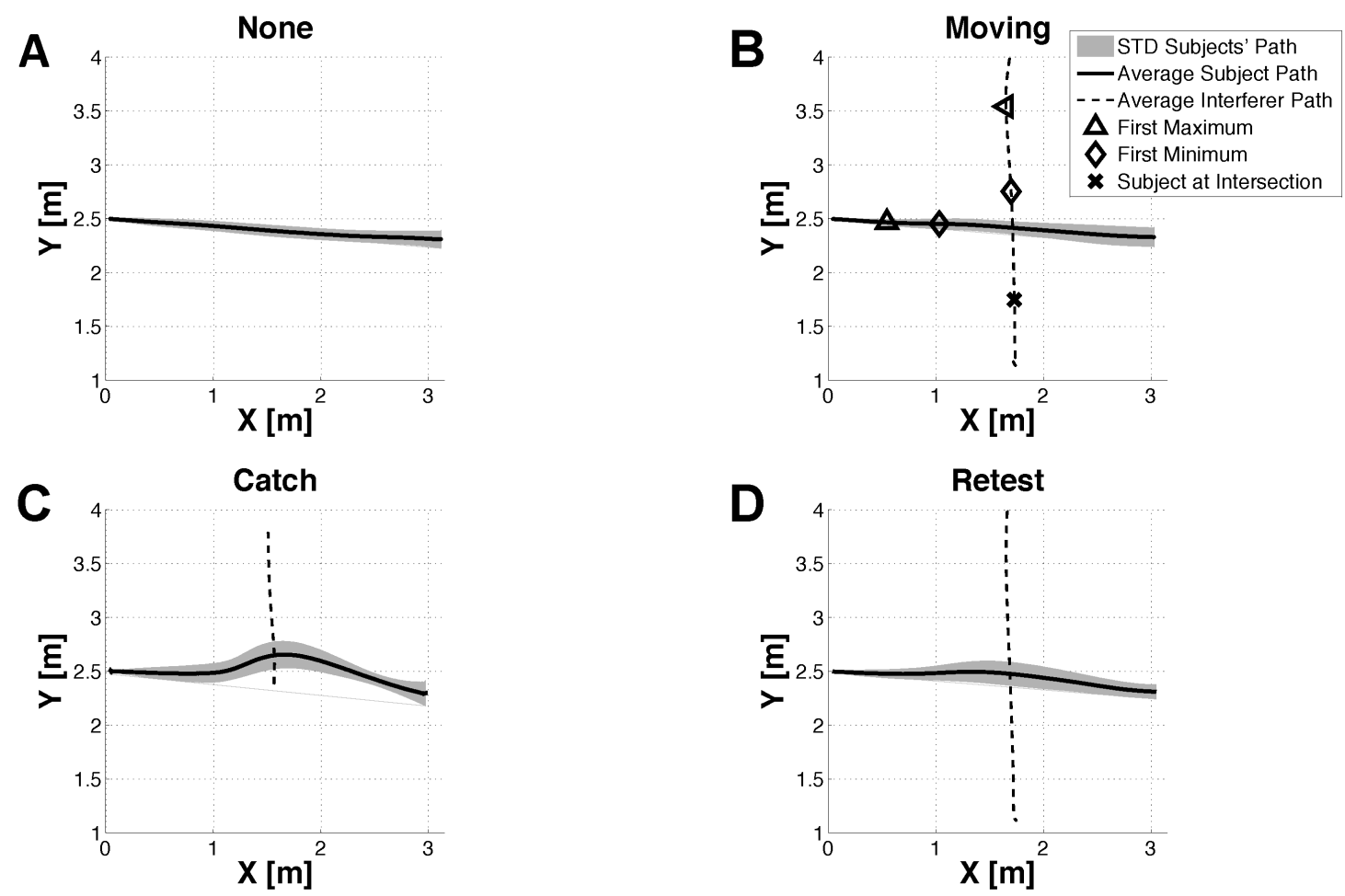

Figure 2: Average path of the subjects for each condition. Shaded area denotes standard deviation of the path. A: Without obstacle. B: Moving obstacle (interferer) condition. The path of the interferer is shown as dashed line. Triangles and diamonds indicate the first maximum and first minimum of the subjects' velocity (see figure $3 \mathrm{~B}$ ) and the average position of the interferer at these points in time. The cross indicates the position of the interferer when the subject passed the intersection point. C: Catch condition. Dashed line shows the interferer's path stopping in the center. D: Retest condition. The moving obstacle condition was repeated twice after the catch trial. 
A

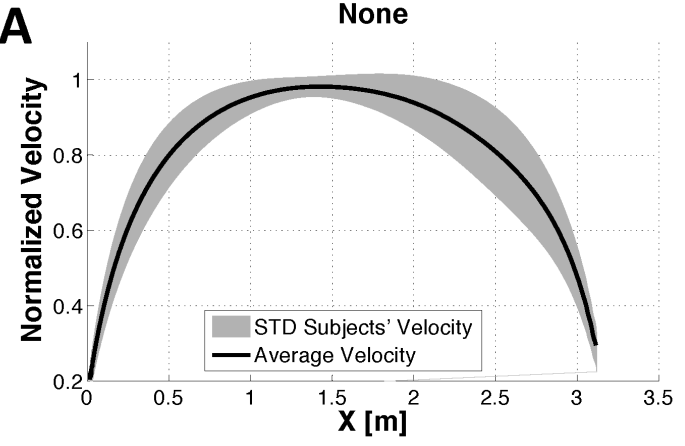

C

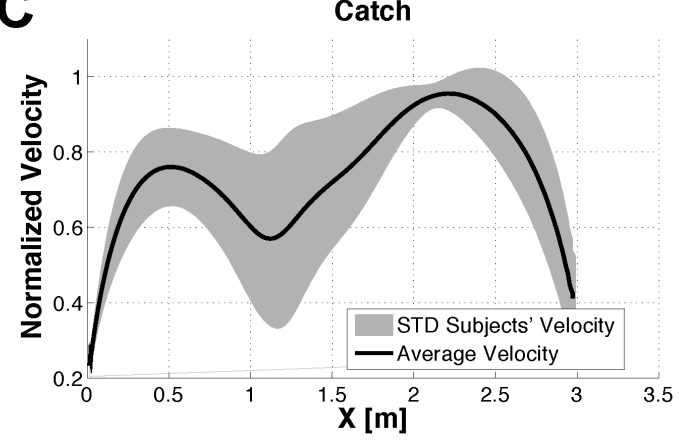

B

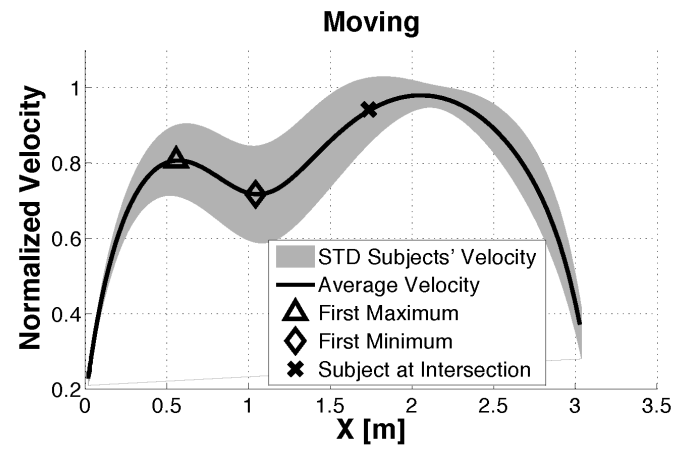

D

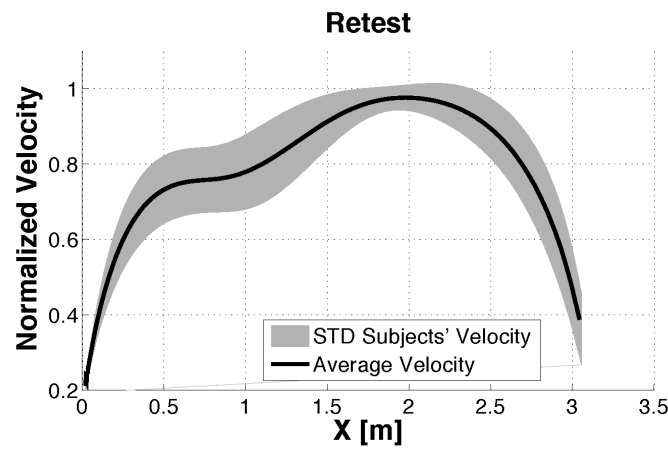

Figure 3: Average normalized tangential velocity profiles plotted over the $x$-coordinate for the conditions presented in Figure 2. The symbols in B correspond to those in Figure $2 B$ with the cross denoting the subject reaching the intersection point in the center.

\section{Simulation of Walking Behavior}

When an appropriate via-point was chosen on the line connecting start and end positions, the simulated behavior that minimized jerk costs closely resembled the observed trajectories (see Figure $4 A_{1}$ ). The velocity and timing of the via-point was selected by minimizing the mean squared error between simulated and observed velocity profile. In order to demonstrate that the observed behavior is not a global minimum-jerk strategy, we simulated a hypothetical alternative strategy where the simulated subject deviates from the straight path (first via-point in Figure $4 \mathrm{~B}_{1}$ ) at around the time when subjects in the Moving condition adapted their velocity (first velocity peak in Figure $4 A_{1}$ ). To pass behind the interferer, a via-point was required (Figure $4 \mathrm{~B}_{2}$ ) that deviated from the straight line between start and end. This alternative strategy turned out to be smoother, i.e., the simulation shown in Fig. 4B had $\sim 3.9$ times lower jerk cost than the simulated movement modeled after the 
subject's behavior (Fig. 4A). Simulation of a static obstacle condition (cf. trajectory shown in [16]) where the subject had to walk around an obstacle located at the center (Figure 4C) also gave a smoother trajectory with $\sim 3.3$ times lower jerk cost.
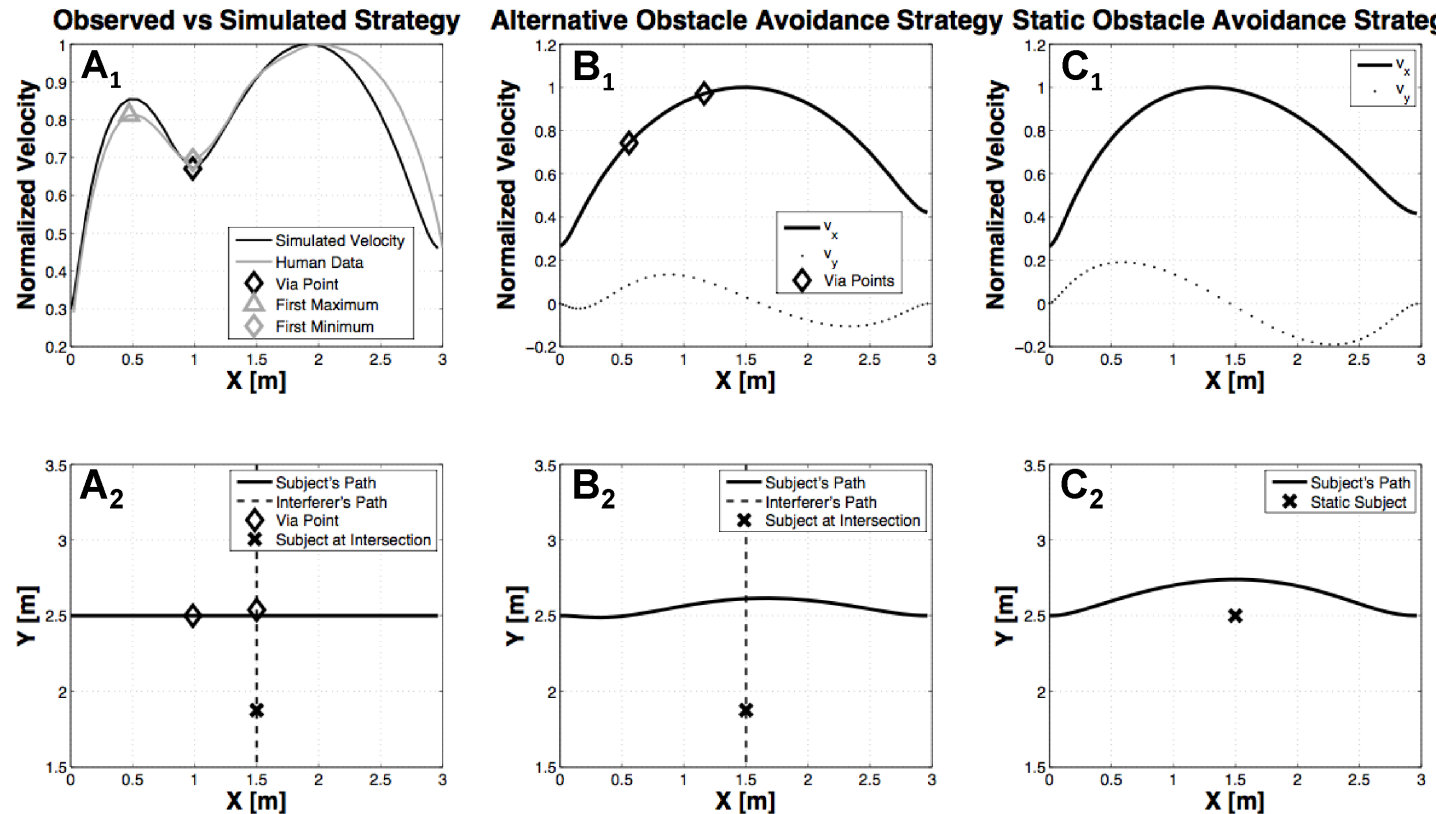

Figure 4: Simulations. $\mathbf{A}_{\mathbf{1}}$ : observed mean velocity (gray) compared with the simulated velocity profile. The via-point for the simulation was chosen such that it corresponded to the observed average velocity of the subjects with minimum mean squared error. The jerk cost for this case is $J=7.92 \mathrm{~m}^{2} / \mathrm{s}^{5} . \mathbf{A}_{2}$ : simulated subject and interferer path with the corresponding via-point position of the subject and interferer (diamond) and interferer's position when the subject crosses the intersection of the paths (cross, c.f. Fig. $2 B$ ). B $_{1}$ : simulation of the velocity, in $x$ - and $y$-axes, of a hypothetical alternative strategy involving path deviation instead of velocity modulation to avoid the moving obstacle. $\boldsymbol{B}_{2}$ : trajectories for the alternative strategy. As in $A_{2}$, the cross corresponds to the interferer's position when the subject is at the intersection of the paths. The jerk cost of this strategy is J=2 $\mathrm{m}^{2} / \mathrm{s}^{5}$. $\boldsymbol{C}_{\mathbf{1}}$ : simulation of the velocity, in $x$-and $y$-axes, of a static obstacle scenario. Here the interferer is placed on a fixed position and the simulated subject is therefore forced to walk around him. $C_{2}$ : simulated trajectories for the static case. The jerk cost for this case is $\mathrm{J}=2.42 \mathrm{~m}^{2} / \mathrm{s}^{5}$.

\section{Discussion}

Human locomotion in the presence of static constraints such as doorways and viapoints has been shown to be stereotyped (e.g., $[5,6,15])$ and to follow a maximumsmoothness constraint [7,8]. Our data confirm the stereotypy of previous locomotion studies for a moving obstacle, in our case another human crossing perpendicularly. However, we show that the maximum-smoothness constraint holds 
only locally, i.e., the chosen straight trajectory was maximally smooth given its path and a particular via-point constraint, but a smoother trajectory always existed that would have allowed to pass without collision. Nonetheless, since, with very few exceptions, subjects generated stereotyped trajectories in each tested scenario and showed no adaptation to or influence of catch trials, a subject-independent strategy underlying obstacle avoidance is likely to exist.

\section{Stereotypy}

In the absence of conflicting moving obstacles, trajectory formation closely followed the maximum smoothness constraint [7]. When this preferred motion would have led to a collision with an interferer, it was modified by a change in velocity profile that allowed subjects to pass behind the interferer at a safe distance. The strategy chosen was very effective: similar to previous results with a $180^{\circ}$ approach [9], there was only one single collision during the whole experiment. Nonetheless, there is a difference between our results for a $90^{\circ}$ approach and those for the $180^{\circ}$ approach $[9,16]$ : while our subjects only changed the velocity profile, an obstacle approaching at $180^{\circ}$ inevitably requires changing the path taken, since any straight line would lead to a collision.

Our subjects initially accelerated as if there was no obstacle on their way to the target position (compare Fig. $2 \mathrm{~A}$ and $2 \mathrm{~B}$ ), even though the interferer was clearly visible from the beginning and the subjects were informed about and could readily infer his walking direction. Again, this behavior was very stereotyped with only one exception: one subject accelerated much slower right from the beginning, but also stayed on the straight path. The strategy chosen by the subjects was not only stereotyped within and between subjects, but also over trials: no adaptation over the four trials could be observed. This suggests that subjects had chosen an 'optimal' strategy right from the beginning, and that repeated observation of the interferer did not bear any new information that could be exploited for further 
optimization. Surprisingly however, the strategy still remained identical after experiencing the catch trial - again with one exception: one subject now walked on a curved path passing behind the interferer, a strategy which accommodates both the 'moving' and the 'catch' conditions. Since the majority of subjects chose the same strategy before and after the catch trial, this particular choice of strategy seems to be very robust, which again points to an underlying general principle governing trajectory formation.

Planning strategies

Assuming a certain cost function such as maximum smoothness does not completely specify the resulting trajectory. For example, planning strategies may differ regarding the use of feedback information: feedback may simply be used as servomechanism trying to follow a pre-planned trajectory, or may take into account the inherent uncertainty about the sensory information [22]. One possible feedforward planning strategy would be to predict the complete time course of the obstacle in advance and to choose an optimal trajectory accordingly. However, such a global planning strategy would result in a curved trajectory with lower cost, as shown in our simulation when the obstacle was placed statically at the intersection point (Fig. 4C). Moreover, as can be seen from the catch trials (Fig. 2C), feedback is available for re-planning of the trajecory [8].

Accordingly, a local planning strategy might be implemented that treats the interferer as static obstacle in every planning step taking into account sensory feedback about the position of the obstacle. In that case, the subject would start out in a straight line until the interferer would cross the currently planned path. At that point, the interferer becomes an obstacle, and the next planning step would inevitably result in a deviation from the straight line. Thus, such a strategy, which closely resembles the one implemented in human-aware robotic path planners $[17,19]$, does also not match the data of the present study. 
A third possible strategy uses local re-planning together with trajectory prediction to continuously estimate the movement of the interferer taking into account the uncertainty about its behavior and to accordingly arrive at an optimal trajectory [22]. We can estimate the uncertainty about the movement of the interferer from the experimental trajectory: our subjects assumed that they could pass behind the interferer more than $0.8 \mathrm{~m}$ before the intersection point, as can be seen by the acceleration of the velocity profile (Fig. $2 \mathrm{~B}$ and $3 \mathrm{~B}$ ). Thus, immediately before this instance, subjects could have chosen a via-point that lies slightly off the straight path behind the interferer, but would let them pass earlier avoiding braking and thus arrive at a smoother trajectory. This scenario is covered by the simulation shown in Fig. 4B and would also lead to a curved trajectory.

Since in each case, a lower cost was possible, we propose that the underlying cost to be minimized is not just global maximum smoothness with respect to translational motion, but that rotational movement adds to the cost. However, more experimental data are required to assess how whole-body rotation affects movement costs.

\section{Towards a common principle}

The present results confirm that locomotor control contains a high degree of flexibility, but for a given situation tends to converge to a single solution. The latter clearly points towards an underlying common principle of trajectory planning. However, as we have shown, maximization of global smoothness does not hold for complex scenarios of trajectory formation such as collision avoidance. One possibility is that the cost associated with trajectory formation also involves wholebody rotations. Other factors, such as the uncertainty of estimating the future trajectory of a moving agent, are likely to affect the choice of the obstacle avoidance strategy as well. Previous work already has shown the importance of sensory feedback for trajectory formation (e.g., vestibular: [23], visual: [24,25]), but, as also demonstrated by our catch trials, it is clear that visual on-line feedback 
constitutes the main modality for recognizing a moving obstacle. Thus any formulation of a common principle governing locomotor trajectory formation has to take into account online feedback mechanisms.

\section{References}

[1] Blake R, Shiffrar M. Perception of human motion. Annu. Rev. Psychol., 2007; 58: 47-73.

[2] Patla AE. Understanding the roles of vision in the control of human locomotion. Gait Posture, 1997; 5: 54-70.

[3] Fajen BR, Warren WH. Behavioral dynamics of steering, obstacle avoidance, and route selection. J. Exp. Psychol. Hum. Percept. Perform., 2003; 29: 343-361.

[4] Gérin-Lajoie M, Richards CL, Fung J, McFadyen BJ. Characteristics of Personal Space during Obstacle Circumvention in Physical and Virtual Environments. Gait , Posture, 2008;27: 239-247.

[5] Hicheur H, Pham QC, Arechavaleta G, Laumond JP, Berthoz A. The formation of trajectories during goal-oriented locomotion in humans. I.A stereotyped behaviour. Eur. J. Neurosci., 2007; 26: 2376-2390.

[6] Pham QC, Berthoz A, Hicheur H. Invariance of locomotor trajectories across visual and gait direction conditions. Exp. Brain Res., 2011; 210: 207-215.

[7] Pham QC, Hicheur H, Arechavaleta G, Laumond JP, Berthoz A. The formation of trajectories during goal-oriented locomotion in humans. II. A maximum smoothness model. Eur. J. Neurosci., 2007; 26: 2391-2403.

[8] Pham QC, Hicheur H. On the open-loop and feedback processes that underlie the formation of trajectories during visual and nonvisual locomotion in humans. J. Neurophysiol., 2009; 102: 2800-2815.

[9] Cinelli ME, Patla AE. Locomotor avoidance behaviours during a visually guided task involving an approaching object. Gait Posture, 2008; 28: 596-601.

[10] Pettré J, Ondřej J, Olivier AH, Cretual A, Donikian S. Experiment-based 
modeling, simulation and validation of interactions between virtual walkers. Proceedings of the 2009 ACM SIGGRAPH/Eurographics Symposium on Computer Animation. 2009; 189-198.

[11] Jacobs A, Shiffrar M. Walking perception by walking observers. J. Exp. Psychol. Hum. Percept. Perform., 2005; 31: 157-169.

[12] Cutting JE, Vishton PM, Braren PA. How we avoid collisions with stationary and moving obstacles. Psychol. Rev., 1995; 102: 627-651.

[13] Berthelon C, Mestre D, Pottier A, Pons R. Is visual anticipation of collision during self-motion related to perceptual style? Acta Psychol 1998; 98: 1-16.

[14] Batty M. Predicting where we walk. Nature 1997; 388: 19-20.

[15] Moussaid M, Helbing D, Garnier S, Johansson A, Combe M, Rohrmüller F, Theraulaz G. Experimental study of the behavioural mechanisms underlying self-organization in human crowds. Proc. Royal Soc. B, 2009; 276: 2755-2762.

[16] Moussaid M, Helbing D, Theraulaz G. How simple rules determine pedestrian behavior and crowd disasters. Proc. Natl. Acad. Sci., 2011; 108: 6884-6888.

[17] Kruse, T., Kirsch, A., Sisbot, E.A. , Alami, R. Exploiting Human Cooperation in Human-Centered Robot Navigation. Proc. 19th IEEE Int. Symp. Robot Human Interactive Communication (RO-MAN), 2010; 212-217

[18] Hall ET. The hidden dimension. New York: Doubleday, Garden City; 1996.

[19] Sisbot EA. Towards human-aware robot motion. Ph.D. Dissertation, Université Paul Sabatier, Toulouse, 2008.

[20] Flash T, Hogan N. The coordination of arm movements: an experimentally confirmed mathematical model. J. Neurosci., 1985; 5: 1688-1703.

[21] Todorov E, Jordan MI. Smoothness maximization along a predefined path accurately predicts the speed profiles of complex arm movements. J. Neurophysiol., 1998; 80: 696-714.

[22] Todorov E. Optimality principles in sensorimotor control. Nat. Neurosci., 2004; 7: 907-915.

[23] Glasauer S, Amorim MA, Viaud-Delmon I, Berthoz A. Differential effects of 
labyrinthine dysfunction on distance and direction during blindfolded walking on a triangular path. Exp. Brain Res., 2002; 145: 489-497.

[24] Warren WH, Kay BA, Zosh WD, Duchon AP, Sahuc S. Optic flow is used to control human walking. Nat. Neurosci., 2001; 4: 213-216.

[25] Wilkie RM, Wann JP. Judgments of path, not heading, guide locomotion. J. Exp. Psychol. Hum. Percept. Perform., 2006; 32: 88-96. 